\title{
"Teatro del oprimido: estrategia didáctica para fomentar el interés por la lectura crítica"
}

Karen Danitza Caballero Cachope*

Artículo de investigación

Fecha de Recepción: 3 mayo 2018.

Fecha de Aprobación: 1 septiembre 2018.

\section{Resumen}

El proyecto de investigación denominado "teatro del oprimido: estrategia didáctica para fomentar el interés por la lectura crítica"se implementó en la sede Saza primaria de la Institución Educativa Saza del municipio de Gámeza - Boyacá. En la formalización del proyecto de investigación se tomaron como muestra a 15 estudiantes de básica primaria, que cursaban los grados de transición hasta quinto, cuyas edades estaban entre los cinco y los once años. Este proyecto de investigación buscó que los estudiantes despertaran agrado por la lectura crítica por medio del teatro del oprimido de Augusto Boal; siendo esta, una experiencia artística que les permitió tener contacto con un material lector diferente al convencional, el cual interiorizaron mediante el desarrollo de ejercicios teatrales orientados por la docente de aula, los cuales facilitaron su libre expresión del cuerpo desarrollando diferentes habilidades de pensamiento crítico y competencias lingüísticas.

Palabras claves: teatro del oprimido, didáctica, pedagogía, lectura crítica, educación rural y ejercicios teatrales.
* Institución Educativa Saza del Municipio de Gámeza -

Boyacá - Colombia pinkdanitza@gmail.com

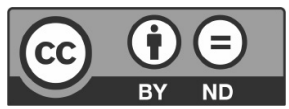




\section{Introducción}

Este proyecto de investigación nació con el propósito de fomentar el gusto por la lectura crítica en los estudiantes de educación básica de la sede Saza primaria del municipio de Gámeza Boyacá, de esta manera mejoraron los resultados de las pruebas saber en el área de lenguaje establecido por el ministerio de Educación de Colombia. Además, la población estudio por medio del teatro desarrolló diferentes habilidades cognitivas y competencias lingüísticas.

Este proceso de investigación se evidenció por observación directa que en el desarrollo de las actividades escolares a los estudiantes no les agradaba el acto de leer y en los resultados de la pruebas Milton Ochoa se diagnosticó un nivel básico en lectura crítica. En investigaciones consultadas la problemática se presentó como resultado de las prácticas educativas tradicionales, por falta de ambientes lectores en sus hogares, entre otras.

En las prácticas educativas del modelo tradicional, leer implicaba copiar y en la gran mayoría de casos, las lecturas eran seleccionadas solo por el docente, el estudiante ocupaba un rol pasivo y su mente se consideraba una tabula rasa. Según Locke (1986), "la mente nace completamente en blanco incluso sin reglas para procesar la información que le llega de los sentidos; todos los datos y las reglas para procesarlos son aprendidos de las experiencias sensoriales. La mente no tiene cosas innatas" (p.83). Este modelo pedagógico, en principio validado, cohíbe al estudiante de la participación activa en las actividades escolares, no valora los saberes previos e interés del educando. La educación rural en Colombia durante años siguió parámetros del modelo tradicional, por consiguiente, es evidente que existieran secuelas de dichas prácticas pedagógicas.

En este proyecto de investigación la experiencia teatral fue la estrategia didáctica que rompió con las prácticas tradicionales, le permitió al estudiante su desenvolvimiento natural en su medio escolar para que desarrollara habilidades cognitivas, emocionales y sociales, que le permitieran tener una participación activa en los proceso educativos. En consecuencia, el material lector fue seleccionado a partir de los intereses de los estudiantes, condición que incentivo al participante acercarse de forma autónoma a este material. Desde esta orientación basándose en la visión de Pennac (2008) quien afirma que: "el miedo a leer se cura con la lectura, el de no comprender con la inmersión en el” (p.2). Se logró que este proceso lector invitara al estudiante a tener acceso al material lector de forma espontánea y libre.

Estudiando las condiciones previas de los hábitos lectores, se observó que la gran mayoría de los estudiantes no cuentan con ambientes lectores en sus hogares. En la vereda Saza donde se encuentra la sede, los acudientes se dedican a la agricultura, la mayoría no alcanzaron a culminar sus estudios de educación básica y otros nunca fueron a la escuela. A esta situación se le sumaba el poco material lector al que tenían acceso los estudiantes. Ante esta 
realidad se solicitó apoyo a la biblioteca municipal y a las bibliotecas virtuales, en donde se descargó material.

En el proyecto de investigación se implementó la investigación acción donde el teatro es una de las estrategias didácticas, que fomentó el gusto por la lectura crítica en los estudiantes de básica primaria. La situación problema describió diferentes eventualidades donde se evidenció que el problema afectaba el desarrollo de las actividades de compresión lectora en todas las áreas del saber. Igualmente en los resultados de las pruebas institucionales y las impuestas por el ministerio de educación se manifestó esta realidad que inspiro la presente investigación. En efecto, con el desarrollo de este proyecto se realizó una transformación social en el contexto escolar, que con la participación activa de la docente y de los estudiantes por medio de ejercicios de sensibilización e introducción al teatro, los participantes tuvieron la oportunidad de leer diferentes obras literarias.

La investigación se centró en el enfoque cualitativo. Acorde con Blasco \& Pérez (2007) quienes señalan que: "la investigación cualitativa estudia la realidad en su contexto natural y cómo sucede, sacando e interpretando fenómenos de acuerdo con las personas implicadas" (p.25). Esta línea de investigación permitió la interacción de la comunidad educativa, la cual por medio del teatro como experiencia artística activo los canales de comunicación y las competencias lingüísticas. Definitivamente con la ejecución de los juegos dramáticos, las dramatizaciones, juego de roles, ejercicios de escritura e improvisaciones los estudiantes experimentaron vivencias significativas que los motivaron a leer de forma libre.

En la proceso de investigación se trabajó técnicas e instrumentos cualitativos para recolectar información (prueba diagnóstica, diario de campo pedagógico, observación directa, prueba final), el paradigma que se implementó fue el socio - crítico, promoviendo la autorreflexión de la docente en su práctica pedagógica y el conocimiento profundo de las necesidades singulares de aprendizaje. La prueba diagnóstica permitió identificar que los estudiantes se encontraban en un nivel básico de lectura crítica, la prueba que se aplicó fue la de Milton Ochoa, la cual tiene un enfoque en la caracterización del nivel de fluidez y comprensión lectora.

Las observaciones directas facilitaron la recolección de información. Según Sabino (1992) "la observación puede definirse, como el uso sistemático de nuestros sentidos en la búsqueda de los datos que necesitamos para resolver un problema de investigación” (p.111). Por tanto, fueron de vital importancia en esta investigación cualitativa permitiendo el registro sistemático, efectivo y confiable de comportamientos, conductas, situaciones de la población objeto de estudio y de la docente investigadora.

El diario de campo pedagógico fue el instrumento donde se registraron todas las observaciones de los acontecimientos, hechos o reflexiones observadas de los ejercicios teatrales,
En el proyecto de investigación se implementó la investigación acción donde el teatro es una de las estrategias didácticas, que fomentó el gusto por la lectura crítica en los estudiantes de básica primaria. 
El estudio de Boal sobre el teatro nació por las dictaduras latinoamericanas. El proceso de formación impartido por este autor tenía como fin orientar a los campesinos brasileros para que lucharan contra las ataduras del discurso de sus opresiones. experiencias que se sistematizaron para ser analizadas. El diario facilitó el desarrollo de procesos de interpretación y análisis crítico de las prácticas teatrales desde una visión constructiva por parte de la docente investigadora que permitió ver los resultados satisfactorios de la implementación de la estrategia didáctica.

La prueba final fue el instrumento que permitió validar la estrategia didáctica de forma global e integral, dando a conocer los alcances de la propuesta en la comunidad educativa, allí se implementaron formatos de cuestionarios donde las preguntas llevaron a los participantes a responder de forma crítica y reflexiva, a partir de sus vivencias, ideas, sentimientos que se despertaron con las prácticas teatrales.

\section{Metodología}

Categorías formativas del teatro: una propuesta para el interés por la lectura

Inicialmente se realizó un análisis reflexivo sobre cada una de las categorías epistemológicas que sustentaron el proyecto de investigación desde una visión teórica del teatro del oprimido. La metodología de autorreflexión direccionó los objetivos de la investigación con el ánimo de fomentar el interés por la lectura desde una perspectiva crítica, por lo anterior, se estudió el teatro como estrategia didáctica, el teatro en la escuela, pedagogía teatral, pedagogía del oprimido de Paulo Freire y el nivel de compresión lectora crítica.

\section{El teatro del oprimido de Augusto Boal}

"El teatro es la propiedad bumana que permite al ser humano verse en acción”. (Augusto Boal, 2002)

El teatro de oprimido brindó herramientas donde los participantes desarrollaron procesos de pensamiento, que los incentivaron a pensar de forma crítica y a debatir un problema empleando el cuerpo. Desde esta mirada Boal (2002) argumenta que "El elemento más importante del teatro es el cuerpo humano; es imposible hacer teatro sin el cuerpo humano" (p.22). Debido a lo anterior, el teatro se convirtió en un dinamizador de la lectura, permitiendo que los estudiantes vivieran experiencias significativas que los motivaron a leer de forma autónoma.

El estudio de Boal sobre el teatro nació por las dictaduras latinoamericanas. El proceso de formación impartido por este autor tenía como fin orientar a los campesinos brasileros para que lucharan contra las ataduras del discurso de sus opresiones. Al respecto Boal (2002) afirma "El Teatro del Oprimido se creó para servir al pueblo, no al revés. Es cierto que al principio tuvimos enemigos muy evidentes a los que podríamos llamar Antagonistas, Opresores: vivíamos en países dominados por tiranos” (p.17).

Los opresores eran el conjunto de razones socio- políticas, los dictadores, leyes que iban en contra de los derechos de los ciudadanos y la discriminación que sufrían los negros y campesinos. De modo que el teatro del oprimido orientó a los campesinos a luchar por sus tierras, 
pero los actores que participaron de las obras teatrales y Boal eran personas de ciudad, presentó una trasformación significativa a partir de una experiencia con trabajadores campesinos de izquierda, por ende esta metodología teatral fue acorde a la población objeto de la investigación que eran campesinos que interactúan en un entorno rural.

El proyecto de investigación se fundamentó en esta metodología basada en el conocimiento del cuerpo y el trabajo colectivo, se organizó bajo los siguientes preceptos:

\section{El teatro foro}

Es un juego que tiene sus propias reglas, la principal es que todos participen activamente y se preste una discusión profunda. Según Boal (2002) lo ideal es que se evite "el foro salvaje, en el que cada uno hace lo que quiere y representa a quien se le ocurra" (p.68). En este espacio los estudiantes defendieron sus ideas y dieron a conocer sus posturas o juicios, el proceso de reflexión no apareció al final de la escena se dio en el desarrollo de toda la obra de teatro.

En conclusión el teatro foro les permitió a los estudiantes en el montaje de las escenas de las obras de teatro liberar su memoria y la imaginación, quienes lograron recordar experiencias vividas y dar juicios para darle solución a una situación en concreto definida por el contenido de las obras literarias.

\section{El juego dramático}

El juego es una herramienta esencial para el proceso de enseñanza, es posible crear reglas concisas y claras que permitieron a los participantes el trabajo colectivo, en esta dinámica es posible crear un ambiente de familiaridad, donde se realizan ejercicios con el cuerpo, esencial para el desarrollo de ejercicios teatrales, desde esta mirada Boal (2002) argumenta 'El elemento más importante del teatro es el cuerpo humano; es imposible hacer teatro sin el cuerpo humano" (p.22).

Los juegos dramáticos permitieron por medio del cuerpo que se promoviera una práctica comunicativa natural, en esta experiencia los estudiantes lograron expresar un lenguaje corporal para crear canales de comunicación, mediante improvisaciones representaron situaciones que luego fueron analizadas e interpretadas colectivamente.

\section{Teatro Imagen}

Esta experiencia artística tiene como propósito utilizar la imagen para que los participantes desarrollen procesos de pensamiento, que los incentivé a pensar de forma crítica y a debatir un problema sin el uso de la palabra, solo empleando el cuerpo. Boal (2002) propone que " los espectadores, como si fuesen escultores, esculpan un grupo de estatuas, es decir, imágenes formadas por los cuerpos de los demás participantes y por objetos encontrados en el local, que revelen visualmente un pensamiento colectivo"(p.41). De este modo se vislumbró una sincronía corporal entre todos los participantes.

Este ejercicio inició con un debate, cada participante tuvo la posibilidad de modificar la estatua inicial, que a partir de sus nociones, percepciones 
y conocimientos demostraron visualmente su postura, finalmente en cámara lenta cada estatua debía actuar como lo haría el personaje que interpretó, demostrando su propia personalidad. Sus movimientos eran los movimientos determinados por las aspiraciones del personaje estatua y no los suyos propios.

De acuerdo con Mouton (2009), el teatro del Oprimido facilita el intercambio de pensamientos e ideas entre los espectadores y actores, lo que permite al público participar para debatir constructivamente sobre una situación o experiencia especifica. En esta experiencia el ejercicio teatral promueve "una serie de actitudes y habilidades personales y colectivas que facilitan la compresión de las problemáticas sociales y en especial se desarrolla la capacidad de escuchar al otro" (Mouton, 2009, p.5). En el teatro del oprimido los participantes tuvieron un espacio teatral para reflexionar, analizar y transformar en escenas diferentes actitudes, comportamientos y situaciones cotidianas.

\section{El teatro como estrategia didáctica}

Referente al teatro como estrategia didáctica, González (2007) de la universidad de Barcelona en su artículo describió las vivencias de los docentes. Desarrolló un taller que según él tenía como propósito:

Motivar al maestro en su práctica docente con un enfoque dinamizador y a la vez a los estudiantes que disfruten de actividades es- colares dinámicas... Desde esta configuración el teatro despierta sentimientos más profundos que facilitan la entrada al mundo del conocimiento y a la transformación de los procesos educativos. (González, 2007, p.49)

El teatro es concebido como una estrategia didáctica puesto que brinda las directrices para que los docentes practiquen procesos creativos en sus actividades escolares y los estudiantes desarrollen habilidades de expresión escrita, corporal y lectora.

Según Motos (2009) en su artículo el teatro en la educación secundaria: fundamentos y retos "el teatro es interdisciplinar es el lenguaje total de todas las asignaturas es un relevador instrumento didáctico eficaz para desarrollar aspectos de las competencias básicas, especialmente: la competencia en comunicación lingüística” (p.9). Desde esta mirada el teatro es la estrategia didáctica que permitió ver, leer, analizar e interpretar material textual, es decir entender y disfrutar la lectura desde un punto de vista crítico y constructivo. Respecto a la formación de los docentes que deseen aplicar esta estrategia, tienen la capacidad de convertirsen en orientadores de expresión artística, porque el docente es un artista innato por su formación en pedagogía.

En esta línea teórica es importante resaltar que en la investigación se evidenció que el teatro desde el enfoquede Augusto Boal es una estrategia didáctica que ofrece al maestro herramientas creativas y lúdicas que incentivan a los estudiantes a leer de forma crítica, 
la metodología de Boal es concreta se realizaron diferentes juegos dramáticos y técnicas teatrales que promovieron el eficaz desarrollo de competencias lingüísticas y comunicativas en los estudiantes, al finalizar la experiencia artística demostraron una disposición hacia lectura crítica.

\section{El teatro en la escuela}

En el estudio de Merchán (2008) especificó que "el teatro es una práctica artística que es, ante todo, una práctica social” (p.96). En efecto la escuela es un espacio propicio donde los estudiantes experimentaron un ambiente lúdico y vivencias artísticas del teatro que les facilitaron una conexión personal y con las personas con las que se relacionan , creó vínculos afectivos y el desarrollo habilidades cognitivas, corporales y sociales que direccionaron una visión del mundo de forma autocritica, por ende los docentes tiene el reto de continuar con la promoción de espacios artísticos dentro de los establecimientos educativos que contribuyen a fortalecer la formación integral de los educandos.

En el proyecto de investigación fue posible crear dentro de la escuela un espacio artístico para desarrollar juegos y técnicas teatrales que les permitieron a los estudiantes vencer sus miedos y aportar todas sus ideas a partir de la lectura de material textual que era analizado minuciosamente para que los participantes se apropiaran de sus personajes y construyeran sus propios libretos. En esta experiencia la escuela rural fue el primer espacio detonante para promover la lectura por medio del arte.

\section{Pedagogía teatral}

En el ámbito de la educación se presenta una variedad de enfoques para potencializar las capacidades y las habilidades de los educandos, desde esta visión Motos (2013 - 2014), afirma que la pedagogía teatral permite que el estudiante tenga una sincronía con los textos, sostiene que es "el teatro es la materia en la que se estudian y valoran los textos, la historia y biografía de los autores"(p.2). En este proceso el teatro no tiene como propósito formar actores sino despertar en el participante conciencia de sí mismo, de los individuos que lo rodean, su principal intención es el crecimiento individual y grupal por medio de juegos teatrales.

Respecto a la educación teatral Merchán (2008) mantiene en su investigación que esta expresión artística presenta elementos significativos que fortalecen el desarrollo de capacidades cognitivas, físicas y axiológicas, por ende su práctica favorece la formación integral del individuo, principalmente en la resolución de problemas y la imaginación. El teatro interviene en procesos de metacognición, el participante tiene la posibilidad de reflexionar sobre sí mismo y los comportamientos de otros individuos.

El proceso metodológico de la investigación recopiló principios de la pedagogía de Paulo Freire pues el teatro del oprimido de Augusto Boal presentó una conexión directa con esta pedagogía, que se ubicó en una línea humanista que fomentaba la educación liberadora y la lectura crítica del mundo,
Respecto a la educación teatral Merchán (2008) mantiene en su investigación que esta expresión artística presenta elementos significativos que fortalecen el desarrollo de capacidades cognitivas, físicas y axiológicas, 
esta corriente pedagógica fundamenta la educación como un acto social que incentivaba al estudiante a preguntarse, estableciendo un dialogo crítico de su realidad internamente y al relacionarse con otros.

\section{Pedagogía del oprimido de Paulo Freire}

La pedagogía del oprimido de Paulo Freire está centrada a una pedagogía humanizada liberadora, que le permitió a los involucrados conocer a fondo las circunstancias que afectan su estilo de vida y asumen un compromiso para transformar esta realidad, este proceso de concientización se da desde una práctica critica, una visualización de las ventajas y privilegios del mundo opresor, por este motivo a esta pedagogía se le categoriza una autentica generosidad humanista.

Esta noción filosófica radica en que la educación bancaria esta direccionad a la dominación, y la problematizadora que se orienta a la educación liberadora facilita el que hacer dialógico, una interacción de los hechos o de la realdad que se experimenta, al respecto Freire (1970) sostiene" la educación problematizadora se hace, así, un esfuerzo permanente a través del cual los hombres van percibiendo, críticamente, como están siendo en el mundo, en el que y con el que están.” (p.96)

La humanización de los hombres se evidencia cuando tienen la capacidad de analizar su realidad críticamente, establecer un dialogo con el medio y sus relaciones, una interacción recíproca, existe una comunicación que le da pie a la educación liberadora, donde los hombres activan sus proceso cognitivos y se cuestionan sobre su situación y los acontecimientos que afectan a otros, en efecto Freire argumenta que la educación problematizadora "es la devolución organizada sistematizada y acrecentada al pueblo de aquellos elementos que este le entrego en forma inestructurada ". (p.113)

La educación según Freire tiene que estar enfocada no solo a entregar conocimiento como lo hace la concepción bancaria, esta práctica de acomodar a los hombres a sus realidad es una visión dominadora, por lo tanto la importancia de luchar por el pueblo por la recuperación de la humanidad robada, el educador tiene el papel de dialogar con el pueblo, definiendo las diferentes posturas o visiones que se presentan en esta comunicación, evitando la imposición de ideales. En esta línea nace la investigación del universo temático del pueblo o el conjunto de sus temas generadores, la metodología de esta investigación implica una dialogicidad de la educación liberadora, el educando se apropia de los temas generadores y toma conciencia de los matices que involucran los temas. Al respecto Freire argumenta que el tema generador es "una concreción, es algo a lo que llegamos a través no solo de la propia experiencia existencial sino también de una reflexión crítica sobre las relaciones hombre - mundo y hombres - hombres, implícitas en las primeras”. (p.119). 


\section{Lectura critica}

Según Valencia \& Osorio (2011), el hábito lector no es innato implica un proceso riguroso en las primero años, cuando el estudiante está dispuesto a conectarse de forma dinámica con la lectura desde una visión del querer y no como un deber, este habito permitió el desarrollo de habilidades cognitivas para observar, comparar, inferir, interpretar y argumentar, el docente tiene que valerse de estrategias didácticas que motiven al estudiante a leer y a tener una experiencia significativa. Al respecto estos investigadores afirman que "la lectura y la comprensión lectora no son habilidades innatas, sino que se van adquiriendo a medida que el individuo crece" (p.11). Por lo tanto la escuela cumple un papel fundamental en el mundo lector, pues es allí donde el niño en sus primeros ciclos aprende el código escrito.

El proyecto de investigación desarrolló fundamentos del teatro de oprimido incentivando la lectura crítica, una mirada analítica del contenido del material textual, es decir los estudiantes tuvieron la oportunidad de desarrollar habilidades de reflexión y capacidades cognitivas que les permitieron argumentar un juicio de valor, este proceso riguroso abrió las sendas de concebir el conocimiento como un instrumento para definir con matices teóricos como se imagina el mundo. Por consiguiente no es leer por leer, se realizó una lectura sistemática donde se interpretó y analizó el rol de los personajes, igualmente las acciones de los mismo y como se podían transformar para inyectar en la escena teatral un toque de originalidad.

En este mismo panorama Benavides \& Sierra (2013), argumentan que:

La comprensión lectora como proceso cognitivo, resalta el papel del desarrollo de los tres niveles de comprensión: literal, inferencial y crítica. Se desarrollan tres habilidades básicas: interpretación, (formarse una opinión, sacar idea central, extraer conclusiones); organización, (establecer secuencias en el texto, resumir generalizar.) y valoración, (identificar relaciones causales, diferenciar lo verdadero de lo falso, captar el sentido de los argumentos del autor), es decir, la aplicación de una lectura crítica. (p.86)

El hábito de leer tiene una intención pedagógica referente a que el lector desarrolle los niveles de compresión lectora que inducen a tener una mirada crítica del contenido del material lector, el teatro del oprimido con su metodología contribuyó a que los estudiantes desarrollaran estos niveles al promover capacidades cognitivas de autorreflexión.

La lectura es un proceso que requiere del desarrollo de capacidades cognitivas y habilidades de pensamiento que les permitieron a los estudiantes el acercamiento progresivo y significativo del texto, en este desafío se estableció fortalecer el nivel de lectura crítica que según Pinzas (1999):

Es cuando el lector tiene la capacidad de introducir las ideas extraídas del texto a diferentes contextos, este nivel implica 
descifrar las temáticas de lo escrito para reputar el contenido de un texto y dar un juicio crítico. En esta experiencia artística los estudiantes alcanzaron a diferenciar un hecho de una opinión, expresar reflexiones frente a una situación determinada, declarando las relaciones que les provocaba un determinado texto y considerando de forma critica la intención del autor.

\section{Compresión lectora}

La compresión lectora es concebida cuando el lector logra desarrollar un pensamiento crítico, en efecto Mejía (2001) sustenta que "La Comprensión: toma cuerpo cuando el lector está en capacidad de reproducir en su propio lenguaje la idea expuesta por el escritor" (p.35).El principio de la lectura crítica es tener la capacidad de dar un juicio de valor se aplica para determinar si la persona logro comprender el contenido del texto, por ende es importante identificar las ideas y acciones principales, escribir un resumen e inferir el propósito del texto, pero en este proceso sistemático los estudiantes asumieron un interés por enriquecer el material lector al reflexionar los sucesos de forma crítica.

Desde esta percepción Mejía (2001) afirma que "la disposición de la lectura, es un factor clave. El entusiasmo incide en la concentración" (p.34). Para que el lector tenga éxito la compresión lectora es esencial la concentración un factor clave que permite centrar la atención en el material lector, para realizar los proceso de pensamiento que facilitaron una visión crítica y reflexiva de las situaciones expuestas en los textos, el equilibrio emocional juega un papel importante puesto que el entusiasmo es visualizado como una estabilidad sentimental que se manifestó cuando el estudiante demostró agrado por el material textual.

En el mismo horizonte la perspectiva teórica de Solé (1992) sostiene que "leer es la interacción entre texto y lector proceso mediante el cual el primero intenta satisfacer los objetivos que guían su lectura” (p.32). El lector tiene que examinar el contenido de los textos y la lectura tiene que estar orientada por un objetivo o propósito que determine que se quiere conseguir con la lectura , de ello depende la interpretación que se realice del contenido, por lo tanto el significado del texto lo dio el estudiante cuando puso a prueba sus conocimientos previos y conjuntamente tuvo claro el objetivo por el que leía, lo que facilitó el desarrollo de las proceso mentales que le permitieron comprender con sentido lo que lee.

En el proceso de investigación de Solé (1992) afirma que "la comprensión lectora es ante todo un proceso de construcción de significados acerca del texto que pretendemos comprender" (p.34). El lector activo es aquel que tiene la capacidad de realizar proceso mentales y entender el significado de los textos, esta activación se presenta con el bagaje experimental, es decir con saberes previos de los estudiantes se logró una conexión con el tema, comprender no implica recitar el contenido ,el educando creó la importancia de lo que lee, al identificar su propósito y 
transcendencia, este ejercicio exigió que el participante creara confianza en sus posibilidades como lector y se sintiera motivado hasta finalizar de la lectura.

La comprensión lectora se puntualiza cuando el lector alcanza constituir de forma global las ideas con sus saberes previos y conocimientos construidos por medio del mundo de la lectura, al respecto Cassany, Luna \& Sanz (1994) sustenta que "Quien aprende a leer eficientemente y lo hace con constancia desarrolla en parte su pensamiento, así que el tratamiento didáctico que se le dé a la lectura repercutirá en los niños de tal manera que los acercará o alejará para siempre de los libros" (p.193). La comprensión de los estudiantes se enlazó con la visión que cada uno tenía de sí mismo y del mundo, es la conexión que el lector estableció con el texto, donde se creó un proceso de relación entre el contenido compartido por el autor con el conocimiento que poseía el lector, de esta manera surgieron nuevos significados.

El teatro es la estrategia didáctica que fomentó la lectura crítica, por lo tanto los estudiantes lograron la comprensión del material textual, ya que implicó que los educandos conocieran a profundidad diferentes obras literarias, las cuales tenían que leer cuidadosamente para organizar, analizar e interpretar los diferentes personajes y situaciones; fue necesario que se conectaran con el relato e interactuarán con ideas, conceptos, teorías y diferentes formas de pensamiento, lo que facilitó que el estudiante estimulara su imaginación, aumentara su vocabulario y tuviera una visión crítica del mundo. El teatro se convirtió así en un dinamizado de la lectura dejando de lado las estrategias tradicionales.

\section{Resultados}

\section{Impacto lector del teatro en la escuela de Saza}

En esta práctica artista los estudiantes desarrollaron competencias de expresión oral, kinésicas, escritura y lectura crítica en cada una de los ejercicios teatrales que les exigía poner a prueba todas sus destrezas y capacidades cognitivas, respecto a la parte emocional se logró que cada estudiante demostraron sus sentimientos antes diferentes situaciones del contexto ,también es de resaltar que fue una práctica divertida donde los participantes en medio de risas disfrutaban el rol del personaje que asumía el compañero y el propio, de igual manera en el momento del maquillaje y montar la escenografía, fue gratificante como la población estudio a medida que se iban desarrollando los ejercicios teatrales exigían y demostraban interés por continuar desarrollando ejercicios de teatro foro ,teatro imagen y juegos dramáticos.

Por lo anterior se evidencio que el equilibrio afectivo es fundamental en el desarrollo de cualquier actividad y exigencia cognitiva ,los estudiantes se gozaron las actividades, este goce facilitó el eficaz cumplimiento del objetivo de esta investigación que se centraba en fomentar el interés de la lectura por medio del teatro, los estudiantes de forma libre realizaron la lectura de obras literarias infantiles como el lobito
El teatro es la estrategia didáctica que fomentó la lectura crítica, por lo tanto los estudiantes lograron la comprensión del material textual, ya que implicó que los educandos conocieran a profundidad diferentes obras literarias, las cuales tenían que leer cuidadosamente para organizar, analizar e interpretar los diferentes personajes y situaciones 
El trabajo en equipo y apoyo moral en el montaje de las obras de teatro fueron claves esenciales para que se desarrollaran de forma espontánea ante actividades institucionales como el día de la familia, el día del idioma y el cumpleaños de

la institución Educativa Saza del municipio de Gámeza, los participaron crearon lazos fuertes de amistad aprendió hacer malo del autor Ian Whybrow, futbol goles y girasoles de Jairo Aníbal Niño, la leyenda oriental del hilo rojo, la leyenda de la cacica Siatoba; el anterior material textual fue seleccionado por los participantes que con el desarrollo de un cuestionario dieron a conocer cuales temáticas eran de su interés, los resultados analizados situaron en el primer lugar las temáticas de los animales, deportes y leyendas.

El trabajo en equipo y apoyo moral en el montaje de las obras de teatro fueron claves esenciales para que se desarrollaran de forma espontánea ante actividades institucionales como el día de la familia, el día del idioma y el cumpleaños de la institución Educativa Saza del municipio de Gámeza, los participaron crearon lazos fuertes de amistad, demostraron un avance progresivo se les veía en las escenas seguros, espontáneos y tenían la capacidad de improvisar en el momento en que se le olvidaban los libretos. Esta vivencia artística fue un constructo grupal desde la redacción de los libretos hasta la lectura en la que se disponían espacio para crear un conversatorios para analizar el material textual y la lectura grupal que les permitió desarrollar la capacidad de escuchar al compañero.

En el desarrollo de la estrategia didáctica los estudiantes y la docente investigadora alcanzaron un nivel de compresión enriquecedor del material textual y de cada una de las vivencias teatrales que les permitió cuestionar de forma constructiva situaciones de la vida cotidiana, acontecimientos históricos y el enriquecimiento de la palabra cuando es plasmada de forma escrita en un papel, conocer la forma de ver el mundo de diferentes autores, esta es una nueva propuesta didáctica que continuara en el desarrollo de las diferentes áreas de saber puesto que sus resultados fueron satisfactorios $\mathrm{y}$ los participantes demostraron interés e iniciativa por continuar con las practicas teatrales.

Esta práctica teatral estaba direccionada a que los estudiantes desarrollaran un pensamiento crítico con una secuencia de ejercicios que implicaron en primer lugar en el conocimiento del cuerpo, para luego expresar por medio de este su sentir, activando los procesos de pensamiento, en esta instancia los actores interactuaron con los espectadores, quienes intervienen en las acciones de los personajes con el propósito de enriquecer el papel del personaje en la escena , así construyeron conjuntamente el libreto, finalmente los participantes gracias a las experiencia teatral desarrollaron la capacidad de discutir o debatir sobre ciertos temas y proponer respecto a situaciones de su vida diaria.

Los ejercicios teatrales desarrollados en el proyecto de investigación están comprendidos en cinco momentos (autoconocimiento, sensibilización, imaginación, creación y reflexión) los cuales abordaron todo el proceso teatral del oprimido de Augusto Boal, estos ejercicios fueron diseñados según las necesidades e intereses de los estudiantes y aprovechando los recursos del contexto. 


\section{Ejercicios teatrales}

\section{Autoconocimiento}

En esta etapa los estudiantes por medio de ejercicios corporales, conectaron su cuerpo para comunicarse con sus compañeros y dieron a conocer su posición frente a diferentes situaciones cotidianas. Esta conexión les permitió reconocer el valor del cuerpo como instrumento de expresión y comunicación.

\section{Sensibilización}

En esta etapa los participantes experimentaron con la ayuda de juegos teatrales el goce que despierta el teatro como una experiencia que les facilitó la expresión espontanea de ideas, pensamientos, miedos e inquietudes. En esta transición reconocieron el teatro como una práctica divertida que les permitió poner a flote todas sus capacidades de expresión y desarrollar habilidades corporales indispensables para la puesta en escena del diferente material lector seleccionado bajo sus intereses.

\section{Imaginación}

En esta etapa los estudiantes realizaron improvisaciones poniendo a prueba su imaginación construyeron escenas de forma autónoma de diferentes situaciones cotidianas que los afectan $y$ reconocieron que acciones son pertinentes para darle una solución, de igual manera argumentaron que circunstancias son de su interés en las obras literarias, en esta experiencia crearon esquemas de montaje y diferentes formas de expresión corporal.

\section{Creación}

En esta etapa los estudiantes motivados por montar una obra de teatro con el material lector, seleccionaron su personaje de interés pusieron en práctica las técnicas de producción y elaboraron sus propios libretos mediante el trabajo colectivo, se organizaron las escenas de las obras de teatro donde cada una de ellas fueron ajustadas por la intervención de todos los participantes, quienes dieron a conocer su punto de vista sobre la personalidad de determinado personaje y cómo era posible actuar frente ciertas circunstancias , las obras literarias fueron adaptadas teniendo en cuenta el juicio de valor de los estudiantes para representarlas en una obra de teatro.

\section{Reflexión}

En esta etapa los estudiantes a través del teatro foro reflexionaron sobre diferentes situaciones o circunstancias que se dieron dentro de las escenas, lograron contextualizar la realidad que emerge la vida de un personaje y a la vez analizaron el impacto que genera la obra literaria en su vida, es decir aprendieron de los diferentes hechos que se desarrolló en el material lector, en esta etapa final el estudiante de forma autónoma argumento el impacto de la obra literaria y de las diferentes escenas de la obra de teatro.

La investigación a través de este proceso continuo, les permitió a los estudiantes vivenciar diferentes etapas del teatro del oprimido, estos ejercicios teatrales fueron idóneos para que los estudiantes con el diferente material lector realizaran juicio de valor, análisis minuciosos 
de las escenas y contextualizaran las diferentes situaciones puestas en escena. El desarrollo de este proyecto permitió la implementación de diferentes espacios lectores como la creación de la ludoteca un lugar de esparcimiento donde los estudiantes interactuaban de forma lúdica con los libros 20 minutos antes de iniciar con su jornada académica.

\section{Conclusiones}

"Nuestra escuela dividida y parcelada exalta la cabeza y descuida las manos, favorece la memoria y descuida el movimiento, valora la inteligencia y esconde el cuerpo" (Heladio Moreno, teatro infantil, p.12).

En el desarrollo de este proyecto se fomentó el placer de leer en diferentes contextos lo que permitió que los estudiantes de básica primaria de la Institución Educativa Saza: sede Saza primaria del municipio de Gámeza Boyacá a través del teatro, desarrollaran habilidades mentales y comunicativas. Este proyecto motivo a los educandos a que hicieran del acto de leer una actividad importante en su diario vivir.

El teatro es un proceso de expresión artística donde los estudiantes lograron analizar minuciosamente las acciones, comportamientos, ideales y vivencias de los personajes de las obras literarias, rescatando las principales características de los personajes que representaron en una obra de teatro. Teniendo en cuenta lo anterior la práctica docente permitió generar un impacto social y la transformación de hábitos de lectura en la población objeto de estudio. Para ello los participantes tuvieron acceso a diferentes libros, luego participaron en actividades escénicas, juegos dramáticos, juego de roles, ejercicios de escritura e improvisaciones desde un enfoque del teatro del oprimido de Augusto Boal donde el principal instrumento es el cuerpo.

El teatro en su máxima exponencía didáctica facilitó la conexión con el arte para la presentación de una obra de teatro (Castillo, 2016), en especial en la puesta en escena donde fue necesario definir una escenografía, vestuario, musicalización y efectos sonoros.En efecto para Mejía (2007) "la práctica emerge como como un lugar en el cual los saberes están en la acción, pero también como un lugar de creación de saberes" (p.12). La práctica pedagógica fue reconocida como la participación activa en los procesos educativos, ejerciendo un detonante en la construcción del conocimiento en el proceso enseñanza - aprendizaje.

En este proceso de investigación la maestra investigadora pudo realizar una autoevaluación de su práctica educativa, que le condescendió conocer con detenimiento los sujetos de estudio, el contexto, las causas y efectos del problema; estas acciones iluminaron el sendero en la aplicación de técnicas y métodos eficaces que garantizaron una orientación didáctica coherente a la realidad del contexto escolar. Por lo tanto la sistematización de las prácticas pedagógicas permitió visualizar el teatro como un componente de alfabetización artístico que incentivó a sus participantes activar su proceso de lectura para expresar sentimientos y sus diversas formas de ver el mundo. 
Los resultados del proyecto de experiencia artística se enfatizó en los investigación fueron significativos para juegos teatrales específicamente el teatro toda la comunidad educativa puesto foro, juegos dramáticos y el teatro que los estudiantes desarrollaron imagen, los cuales incentivaron a los competencias lingüísticas por medio participantes a leer de forma autónoma del teatro creando una conexión directa y con agrado. con diferente material textual. Esta

\section{Referencias}

Benavides, D \& Sierra, G. (2013).Estrategias didácticas para fomentar la lectura crítica desde la perspectiva de la transversalidad REICE. (pp. 79-109). Revista Iberoamericana sobre Calidad, Eficacia y Cambio en Educación.

Blasco, E. \& Pérez, A. (2007). Metodologías de investigación en las ciencias de la actividad física y el deporte: ampliando horizontes. Editorial Club Universitario. España.

Boal, A. (2002). Juegos para actores y no actores. Teatro del oprimido. (pp.1 - 389). Alba Editorial .Edición Febrero. Impreso en España.

Cassany, D., Luna, M. \& Sanz, G. (1994). Enseñar lengua. (pp. 1- 576). Barcelona: Editorial Grao.

Castillo, A. (2016). La virgen barroca y las prácticas artísticas en América Latina. Cuestiones De Filosofía, (16), 89-95. https://doi.org/10.19053/01235095.3626

González, M. (2007). El teatro como estrategia didáctica. (pp. 49-55). Universidad de Barcelona.

Locke, J. (1986).Pensamientos sobre la Educación. (pp. 262-263).

Mejía, C. (2001). Apuntes de un investigador. (pp. 1 - 247) www.colombiaaprende.edu. co/html/.../articles- 169771_archivo.doc.

Mejía, R. (2007) .La sistematización como proceso investigativo o la búsqueda de la episteme de las prácticas. (pp.1 - 17).Planeta Paz Expedición Pedagógica Nacional Programa Ondas de Colciencias.

Merchán, C. (2008). La gestión cultural de la educación artística desde las artes escénicas se inicia en el emplazamiento de procesos de formación que devienen de los maestros de las artes escénicas en la escuela básica y media. (pp. 93-107). Segunda época.

Moreno, H. (1993) .Teatro infantil para la enseñanza primaria .Teatro juvenil. Ed. Magisterio, Colombia nueva. Bogotá. 
Motos, T. (2009) .El teatro en la educación secundaria: fundamentos y retos. Creatividad y Teatro. (pp. 1 - 35). Revista Creatividad y Sociedad.

Motos, T. (2013-2014). Psicopedagogía de la Dramatización.( pp. 1 - 36).Universidad de Valencia.

Mouton, S. (2009).Convivir con teatro. El Teatro del Oprimido como herramienta para la elaboración conjunta de la Convivencia Escolar. Primer premio de buenas prácticas para el impulso y la mejora de la convivencia. ( pp 1-20). Ministerio de Educación.

Pennac, D. (2008) Mal de escuela. (pp. 1-172). Literatura Mondadori: Barcelona.

Pinzás, J. (1999). Importancia de la investigación aplicada: reflexiones en relación a la comprensión de lectora. (pp.1-13). Educación. Pontificia Universidad Católica del Perú, Departamento de Humanidades.

Sabino, C. (1992). El proceso de la investigación. (pp. 1 - 216). Editorial Panapo. Caracas, Venezuela.

Solé, P. (1992). Estrategias de lectura. (pp.1 - 199). Editorial Graó. Barcelona. Primera edición.

Valencia, C., \& Osorio, D. (2011) .Estrategias para fomentar el gusto y el bábito de la lectura en primer ciclo. (pp. 1 - 132). Universidad libre facultad de ciencias de la educación, departamento de lenguas, programa de licenciatura en educación básica con énfasis en humanidades e idiomas Bogotá. 